\title{
Criminologie
}

\section{Femmes au-delà des murs : le sens de la quête d'emploi chez les femmes judiciarisées et les intervenants au Québec}

\section{Véronique Strimelle et Sylvie Frigon}

Volume 40, numéro 2, automne 2007

Peines et pénalité au Canada. Autour des travaux de Pierre Landreville

URI : https://id.erudit.org/iderudit/016859ar

DOI : https://doi.org/10.7202/016859ar

Aller au sommaire du numéro

Éditeur(s)

Les Presses de l'Université de Montréal

ISSN

0316-0041 (imprimé)

1492-1367 (numérique)

Découvrir la revue

Citer cet article

Strimelle, V. \& Frigon, S. (2007). Femmes au-delà des murs : le sens de la quête d'emploi chez les femmes judiciarisées et les intervenants au Québec.

Criminologie, 40(2), 167-189. https://doi.org/10.7202/016859ar
Résumé de l'article

Sur la base d'une enquête de terrain menée auprès de femmes judiciarisées au Québec et d'intervenants travaillant avec elles, nous explorons dans cet article la question du travail des femmes judiciarisées après l'emprisonnement. Nous voulons plus spécifiquement préciser comment se pose la question de l'emploi pour ces femmes et le sens qu'elles lui accordent. Nous voulons aussi interroger les ressources existantes en matière d'aide à l'emploi. Sont-elles suffisantes et répondent-elles aux besoins des femmes ? Cette analyse nous conduira ainsi à identifier les ressources, projets et changements souhaités dans l'approche préconisée auprès des femmes judiciarisées pour enfin nous interroger plus globalement sur l'efficacité des formes d'intervention pénale auprès des femmes et la persistance d'une logique carcérale qui continue à s'imposer malgré les multiples échecs qu'on lui reconnaît. En ce sens, notre recherche souligne la nécessité de repenser les formes d'intervention auprès des femmes judiciarisées en matière d'emploi, en prêtant attention à la singularité de leurs expériences et de leurs besoins en matière d'emploi, en tenant compte du contexte structurel d'oppression qu'elles subissent, en essayant enfin d'atténuer, voire de supprimer l'idéologie répressive et les effets négatifs que celle-ci peut avoir sur leurs possibilités de réinsertion. 


\title{
Femmes au-delà des murs: le sens de la quête d'emploi chez les femmes judiciarisées et les intervenants au Québec
}

\author{
Véronique Strimelle \\ Professeure \\ Département de criminologie, Université d'Ottawa \\ vstrimel@uottawa.ca \\ Sylvie Frigon \\ Professeure \\ Département de criminologie, Université d'Ottawa \\ sfrigon@uottawa.ca
}

RÉSUMÉ • Sur la base d'une enquête de terrain menée auprès de femmes judiciarisées au Québec et d'intervenants travaillant avec elles, nous explorons dans cet article la question du travail des femmes judiciarisées après l'emprisonnement. Nous voulons plus spécifiquement préciser comment se pose la question de l'emploi pour ces femmes et le sens qu'elles lui accordent. Nous voulons aussi interroger les ressources existantes en matière d'aide à l'emploi. Sont-elles suffisantes et répondent-elles aux besoins des femmes? Cette analyse nous conduira ainsi à identifier les ressources, projets et changements souhaités dans l'approche préconisée auprès des femmes judiciarisées pour enfin nous interroger plus globalement sur l'efficacité des formes d'intervention pénale auprès des femmes et la persistance d'une logique carcérale qui continue à s'imposer malgré les multiples échecs qu'on lui reconnaît. En ce sens, notre recherche souligne la nécessité de repenser les formes d'intervention auprès des femmes judiciarisées en matière d'emploi, en prêtant attention à la singularité de leurs expériences et de leurs besoins en matière d'emploi, en tenant compte du contexte structurel d'oppression qu'elles subissent, en essayant enfin d'atténuer, voire de supprimer l'idéologie répressive et les effets négatifs que celleci peut avoir sur leurs possibilités de réinsertion.

ABSTRACT - In this article, based on a field research with criminalized women in Quebec and with front line workers, we address the question of criminalized women's work. More specifically, we want to precise how those women define and give meaning to the 
concept of employment. We also want to review some of the existing resources created to help those women to find a job. Are they sufficient and do they really address the needs of the criminalized women? We also present the resources, projects and changes which would positively improve the employment policies toward criminalized women. Finally, we will question the efficiency of the penal policies toward criminalized women. Despite their obvious failures, those policies are still maintained. In that sense, our research emphasizes the need to re-think those policies by considering the women's expectations and experiments in terms of employment, by taking into account the numerous forms of oppression they are still submitted to, by trying to diminish or suppress the structural repressive ideology that negatively impacts on their lives.

\section{Introduction}

Je demande juste un toit sur la tête, trois repas par jour puis un petit emploi stable [...]. Je vais être heureuse avec ça, moi. Commencer à vivre la vraie vie un peu, pas toujours être enfermée en arrière de mes stores, non, non merci. Je veux vivre, de jour surtout, je veux voir le soleil, je suis fatiguée de vivre la nuit. (Cindy, incarcérée en prison provinciale, 37 ans)

Depuis ses origines, la prison a été présentée par ses promoteurs comme un des outils les plus efficaces pour réformer les détenus et en faire des citoyens responsables à leur sortie (Foucault, 1975; Garland, 1985). Depuis lors, l'efficacité de l'enfermement a fait l'objet de nombreuses critiques et remises en question, les détracteurs du modèle pénitentiaire arguant notamment du fait que la prison pouvait être considérée davantage comme une "école du crime» que comme un outil de réinsertion sociale (Lalande, 2000).

Dans la société occidentale, le travail est présenté comme un élément central dans la vie des gens, non seulement parce qu'il leur permet de survivre matériellement, mais aussi parce qu'il leur assure une certaine reconnaissance sociale (Castel, 1995; Gillis, 1996). Cette importance accordée au travail comme facteur de réintégration sociale s'applique aussi dans les milieux correctionnels, le travail y est en effet présenté comme une des voies privilégiées pour retrouver une place dans la société mais aussi comme un moyen de réduire plus efficacement le taux de récidive (Poupart et al., 1989; Gillis, 2000; Brouillard et Sirois, 1996; Strimelle et Poupart, 2004). Comme le suggère Combessie: «[L]e travail pénitentiaire donnerait du sens à l'enfermement» (Combessie, 2005 : 98). 
Actuellement, un des mandats explicite des services correctionnels canadiens est d'aider à la réhabilitation sociale des détenus tout en exerçant sur eux un contrôle en vue de protéger la société du danger éventuel qu'ils peuvent représenter une fois sortis. Ce double mandat de contrôle et de réintégration n'est pas sans effets pour les détenus qui sortent de prison. Obligés de trouver du travail après une incarcération plus ou moins longue, ils ont aussi à composer avec les exigences et la supervision du service correctionnel. Mais le passage en prison ne fait pas que les couper plus ou moins longtemps du milieu du travail, il les intègre aussi à une catégorie sociale marquée par la stigmatisation, ce qui peut entraver toute tentative de réinsertion par le travail (Schmitz, 1985 ; Faugeron, 1996). Se fondant sur les écrits de Fauconnet, Combessie (2004) soutient à ce propos que la permanence de ce stigmate est liée à la fonction sacrificielle de la peine présente dans le système de justice pénale. Suivant cette logique, la sanction pénale est plus dirigée vers le crime commis que vers le criminel lui-même. Comme il est impossible d'annuler l'acte commis dans le passé, le responsable de cet acte fait alors office de «substitut du crime» qui doit être définitivement mis au ban de la société des «honnêtes gens». «Jamais pardonné - ou très exceptionnellement -, le détenu reste 'comme enfermé dans un acte unique'» (Combessie, 2004: 261).

Provenant en général de milieux où l'accès aux ressources économiques, sociales, culturelles est limité, de nombreux détenus et détenues qui sortent de prison ne disposent pas non plus d'outils ou de « réseaux extérieurs de protection rapprochée» qui peuvent les aider à s'en sortir sans sombrer (Faugeron, 1996).

Pour les personnes ayant vécu une expérience d'incarcération, trouver ou retrouver un emploi semble donc quasi impossible. C'est particulièrement le cas pour les femmes qui sortent de prison. Les études faites à leur sujet soulignent que nombre d'entre elles proviennent d'un environnement social défavorisé, criminalisé et qu'elles ont connu un passé marqué d'abus auquel viennent souvent se greffer des problèmes de toxicomanie. Peu scolarisées, elles ont aussi en général exercé peu d'emplois réguliers avant leur incarcération et doivent souvent assumer seules les responsabilités familiales (Blanchard, 2002; Frigon et al., 2003 ; Richie, 2001).

C'est à cette question du retour au travail des femmes judiciarisées que notre article est consacré. Nous voulons mieux comprendre comment se pose la question de l'emploi pour elles et le sens qu'elles lui 
accordent. Voient-elles dans le fait de trouver un emploi ou de garder un emploi une condition indispensable pour ne pas «retomber» dans la délinquance et se retrouver en prison? L'intégration sociale passet-elle nécessairement pour elles par l'obtention d'un emploi? Nous voulons aussi interroger les ressources existantes en matière d'aide à l'emploi pour les femmes: sont-elles suffisantes et répondent-elles à leurs besoins?

Au Canada, au cours des vingt dernières années, la proportion de femmes incarcérées est passée de 5 à $9 \%$ dans les établissements d'incarcération provinciaux et territoriaux et de 3 à $5 \%$ dans les établissements fédéraux où les femmes sont condamnées à des peines de deux ans et plus (Frigon et al., 2003). Malgré cette augmentation, les femmes constituent encore une minorité au sein du monde carcéral (Bertrand, 2002 ; 2003). En 1990, la publication du rapport d'étude La création de choix a contribué à l'émergence de nouveaux discours et de nouvelles formes de traitement pénal des femmes judiciarisées (Frigon, 2002). Malgré ces transformations, la situation et les besoins des femmes détenues restent cependant encore souvent méconnus, voire négligés, particulièrement en ce qui concerne la question de l'accès à l'emploi après la sortie de prison (Bloom, 1999; Gillis, 2000; Combessie, $\left.2005^{1}\right)$.

Outre les écrits féministes relatifs à l'emprisonnement des femmes (Carlen, 1983; Faith, 2002; Hannah-Moffat, 2001; Bertrand, 2002; Frigon, 2004; Buck, 2004) qui ont souligné l'importance d'analyser le vécu des femmes criminalisées et leurs propos en tenant compte du contexte d'oppression dont elles font l'objet, notre démarche s'inspire aussi des travaux portant sur les processus de marginalisation et d'exclusion, notamment ceux de Castel (1994; 1995), de Paugam (1991; 1993), de Laberge et Roy (1994) et de Schnapper (1997). Pour ces auteurs, la question de l'intégration est le résultat de processus complexes dont il faut tenir compte dans l'élaboration de programmes d'aide aux populations plus vulnérables, particulièrement en matière de retour à l'emploi.

1. Cette étude fait partie d'une étude européenne menée dans six pays: l'Espagne, l'Allemagne, la Grande-Bretagne, l'Italie, la France et la Hongrie. 


\section{Méthodologie}

Basée sur une méthodologie qualitative, l'enquête de terrain fut réalisée à la suite d'entrevues semi-directives auprès de vingt et une personnes incarcérées dans des pénitenciers fédéraux et des prisons provinciales ou résidant dans des maisons de transition ainsi qu'auprès de quatorze intervenants travaillant avec les femmes judiciarisées. Dans le cadre de l'enquête, nous avons aussi organisé deux groupes de discussion (focus groups) réunissant douze intervenants d'établissements de détention, de maisons de transition, des services correctionnels provinciaux et fédéraux et d'organismes spécialisés en développement de la main-d'œuvre.

Les questions que nous désirions aborder au cours des entrevues différaient selon le groupe auquel les personnes appartenaient. Pour les femmes judiciarisées, les principaux thèmes abordés concernaient leur situation actuelle, leurs expériences en termes de travail, les programmes suivis en détention, leurs projets en matière d'emploi à la sortie. Pour les intervenants, les questions portaient sur leurs rapports avec la clientèle, leurs conditions de travail, les éléments positifs et négatifs identifiés dans le cadre de leur intervention, les besoins des femmes et les solutions préconisées pour améliorer l'accès à l'emploi.

Un premier échantillon de dix entrevues a fait l'objet d'une lecture préliminaire permettant de dégager les thèmes principaux traités par les femmes. Ces thèmes peuvent être regroupés sous trois grands axes: 1) la situation des femmes; 2) les enjeux de l'intervention auprès des femmes judiciarisées; 3 ) le maintien en emploi et les pistes prometteuses. Par la suite, le reste des entrevues a été lu, annoté et résumé à l'aide de mémos analytiques individuels où les propos des divers acteurs interviewés étaient repris et répertoriés pour chaque grand axe d'analyse. La dernière étape de l'analyse (horizontale) a consisté à reprendre les propos des personnes, à les sélectionner selon leur pertinence et à les présenter en les commentant.

Les groupes de discussion ont eu lieu à deux reprises et regroupaient les mêmes participants. Durant ces rencontres, nous avons pu échanger avec les intervenants sur les enjeux de leur intervention auprès des femmes judiciarisées, les caractéristiques des clientèles, leurs besoins spécifiques en termes d'emplois et les difficultés de la préparation à la sortie et du suivi post-carcéral. L'échantillon principal sur lequel nous avons centré notre attention est celui des femmes, mais les entrevues et les échanges avec les intervenants nous ont permis de clarifier certaines 
questions relatives aux politiques d'aide à l'emploi des personnes judiciarisées, de préciser certains thèmes d'analyse et de vérifier auprès d'eux la pertinence de certaines de nos questions ainsi que de nos résultats.

Compte tenu du fait qu'il nous était impossible pour des raisons légales d'aborder directement des personnes se trouvant sous la tutelle du système correctionnel fédéral et provincial, nous avons d'abord dû obtenir l'autorisation de mener notre recherche auprès des services concernés. Par la suite, plusieurs personnes ressources présentes dans les différentes institutions correctionnelles ont assuré le premier contact avec les femmes judiciarisées et les ont invitées à participer volontairement à la recherche. Si les femmes se montraient intéressées à participer à la recherche, avec leur accord préalable, nous fixions un rendez-vous afin de réaliser l'entrevue.

N'ayant pas de pouvoir réel dans le choix de l'échantillon, nous avons bien conscience de certaines de ses limites. Nous n'avons par exemple pas pu rencontrer de femmes autochtones, surreprésentées dans le système correctionnel (un quart de la population des femmes incarcérées au palier fédéral est autochtone ${ }^{2}$ ), ce qui ne nous a pas permis d'identifier les enjeux spécifiques que ces femmes ont à affronter lors de leur sortie de prison.

\section{Description de l'échantillon}

De manière schématique, le profil des femmes interviewées se présente comme suit: vingt et une femmes contrevenantes ont été interviewées (quatre sous juridiction fédérale et douze sous juridiction provinciale; cinq sous surveillance en maison de transition).

Les femmes rencontrées avaient entre 23 et 55 ans au moment des entrevues. Douze d'entre elles avaient entre 35 et 44 ans et huit avaient entre 35 et 38 ans. Dix-sept d'entre elles ont dit avoir des enfants. Quatorze étaient célibataires; quatre vivaient en couple; trois n'ont pas précisé leur statut, mais d'après leurs propos, n'ont pas de conjoint ou de conjointe. Onze femmes ont dit avoir atteint la cinquième année du secondaire (fin du cycle secondaire). Parmi elles, trois ont suivi des cours au cégep (niveau pré-universitaire) et deux ont suivi des cours à l'uni-

2. Cyrenne (2002: 32). Au Canada, le pourcentage de femmes autochtones vivant dans la pauvreté représente plus du double de celui des femmes non autochtones (Towson, 2005: 2). 
versité. Pour sept autres femmes, le niveau de scolarité atteint allait de la première à la quatrième année du secondaire. Enfin, trois femmes n'ont pas précisé leur niveau de scolarité. Les sentences encourues variaient entre trois et soixante mois et une femme était prévenue au moment de l'entrevue, donc en attente de jugement. Huit femmes étaient incarcérées pour vol, vol à l'étalage ou fraude ${ }^{3}$; trois pour des bris de conditions; deux pour des délits reliés aux drogues; deux pour voies de fait et menaces de mort, et deux pour sollicitation et non-dénonciation. Trois femmes étaient aussi emprisonnées pour homicide (deux meurtres et un homicide involontaire). Celles qui étaient condamnées pour meurtre subissaient des peines d'emprisonnement à perpétuité. L'une d'entre elles avait interjeté appel et l'autre poursuivait sa peine à l'extérieur ${ }^{4}$. Treize femmes subissaient une sentence de moins de vingt mois, cinq femmes purgeaient des peines allant de trente à soixante mois d'emprisonnement. En ce qui concerne les intervenants rencontrés lors des groupes de discussion et en entrevue, la majorité d'entre eux étaient des femmes, un seul homme était représenté parmi les interviewés et un seul homme présent lors des deux séances de groupes de discussion.

Dans les pages qui suivent, nous allons explorer les thèmes qui ont émergé à la suite de l'enquête de terrain. Nous avons choisi de présenter les données en découpant notre matériel suivant les trois axes d'analyse déjà cités: 1) les femmes judiciarisées et l'emploi; 2) les défis de l'intervention en emploi auprès des femmes judiciarisées; 3) les pistes prometteuses.

\section{Les femmes judiciarisées et l'emploi}

Situation d'emploi des femmes rencontrées

Le tableau 1 indique la situation de ces femmes en matière d'expériences d'emploi :

3. En 2000, pour la population des femmes sous juridiction provinciale, les principaux délits pour lesquels les femmes étaient détenues étaient la conduite avec facultés affaiblies, la sollicitation et le vol à l'étalage (Comité aviseur pour la clientèle judiciarisée adulte, 2001).

4. Rappelons ici que les personnes condamnées à perpétuité restent continuellement sous la tutelle du service correctionnel, même après avoir quitté le pénitencier. 
TABLEAU 1

Sources de revenus et expériences en matière d'emploi

\begin{tabular}{|c|c|c|c|c|c|}
\hline Nom fictif & $\begin{array}{l}\text { Emploi légal avant } \\
\text { détention }\end{array}$ & $\begin{array}{l}\text { Emploi } \\
\text { illégal }\end{array}$ & $\begin{array}{l}\text { Assistance- } \\
\text { emploi }\end{array}$ & $\begin{array}{l}\text { Assurance- } \\
\text { emploi }\end{array}$ & $\begin{array}{l}\text { Emplois en } \\
\text { détention }\end{array}$ \\
\hline Anabelle & $\begin{array}{l}\text { Serveuse, femme de } \\
\text { chambre, réceptionniste, } \\
\text { agente de sécurité }\end{array}$ & Oui & Non & Non & $\begin{array}{l}\text { Buanderie, } \\
\text { cuisine, ménage }\end{array}$ \\
\hline Angela & Infirmière & Non & Non & Non & $\begin{array}{l}\text { Alimentation, } \\
\text { ménage }\end{array}$ \\
\hline Anne & $\begin{array}{l}\text { Travail de bureau et } \\
\text { informatique, caissière }\end{array}$ & Non & Non & Non & $\begin{array}{l}\text { Cuisine, } \\
\text { alimentation }\end{array}$ \\
\hline Catherine & $\begin{array}{l}\text { Secrétaire-réception- } \\
\text { niste, réception- } \\
\text { expédition }\end{array}$ & Oui & Oui & Non & $\begin{array}{l}\text { Buanderie, } \\
\text { ensachage, } \\
\text { ménage }\end{array}$ \\
\hline Christina & $\begin{array}{l}\text { Secrétaire- } \\
\text { réceptionniste }\end{array}$ & Oui & Non & Oui & $\begin{array}{l}\text { Nettoyage de } \\
\text { téléphones et } \\
\text { de chandeliers }\end{array}$ \\
\hline Cindy & $\begin{array}{l}\text { Gardienne d'enfants, } \\
\text { manufacture, barmaid, } \\
\text { danseuse }\end{array}$ & Oui & Oui & Non & $\begin{array}{l}\text { Buanderie et } \\
\text { cuisine }\end{array}$ \\
\hline Édith & Cuisine, barmaid & Oui & Oui & Non & Buanderie \\
\hline Héloïse & $\begin{array}{l}\text { Auxiliaire pour pers. } \\
\text { âgées, contremaître, } \\
\text { secrétaire, caissière, } \\
\text { serveuse }\end{array}$ & Non & Inconnu & Inconnu & Buanderie \\
\hline Isabelle & $\begin{array}{l}\text { Barmaid, commis, } \\
\text { télémarketing, } \\
\text { répartitrice et vente } \\
\text { sur route, vendeuse }\end{array}$ & Oui & Non & Oui & $\begin{array}{l}\text { Bibliothèque, } \\
\text { cuisine, } \\
\text { intervenante } \\
\text { centre de } \\
\text { femmes }\end{array}$ \\
\hline Janet & Secrétaire, ménage & Non & Oui & Non & Ensachage \\
\hline Jannika & $\begin{array}{l}\text { Serveuse, commis, } \\
\text { technicienne pose } \\
\text { d'ongles, manufacture }\end{array}$ & Non & Non & $\begin{array}{l}\text { Oui, } \\
\text { saisonnier }\end{array}$ & $\begin{array}{l}\text { Buanderie, } \\
\text { ensachage }\end{array}$ \\
\hline Josée & Caissière (banque) & Inconnu & Inconnu & Inconnu & Animation \\
\hline Linda & Caissière (magasin) & Oui & Oui & Non & $\begin{array}{l}\text { Imprimerie, } \\
\text { couture, cuisine }\end{array}$ \\
\hline Louise & Aucun & Non & Oui & Non & $\begin{array}{l}\text { Ateliers, } \\
\text { ensachage }\end{array}$ \\
\hline Malika & Danseuse & Oui & Oui & Non & $\begin{array}{l}\text { Garderie, } \\
\text { alimentation }\end{array}$ \\
\hline Marie-Lou & $\begin{array}{l}\text { Vendeuse, serveuse, } \\
\text { cuisinière, gérante } \\
\text { casse-croûte, ménage, } \\
\text { comptabilité, danseuse }\end{array}$ & Inconnu & Oui & Non & Bibliothèque \\
\hline Marie-Sable & Informatique & Non & Oui & Oui & Non \\
\hline Mélanie & $\begin{array}{l}\text { Serveuse, caissière, } \\
\text { travail agricole, } \\
\text { danseuse }\end{array}$ & Oui & Inconnu & Inconnu & Non \\
\hline Roxy & Danseuse & Oui & Oui & Non & Non \\
\hline Sheila & $\begin{array}{l}\text { Agro-alimentaire, } \\
\text { restaurant, } \\
\text { mécanicienne }\end{array}$ & Oui & Oui & Non & $\begin{array}{l}\text { Cuisine, } \\
\text { imprimerie }\end{array}$ \\
\hline Suzanne & $\begin{array}{l}\text { Cuisine, aide aux } \\
\text { bénéficiaires }\end{array}$ & Oui & Oui & Inconnu & Cuisine \\
\hline
\end{tabular}


Comme on le voit, toutes les femmes avaient déjà occupé un travail légal à un moment ou l'autre de leur vie, la très grande majorité ont mentionné avoir exercé des emplois illégaux avant leur incarcération. Certaines femmes ont aussi bénéficié de revenus provenant des programmes d'assistance-emploi et/ou d'assurance-emploi avant leur incarcération (rappelons ici que l'assistance-emploi est un programme fédéral d'aide financière destiné aux personnes privées de leurs moyens de subsistance, alors que l'assurance-emploi fournit une assurance financière temporaire aux chômeurs, aux travailleurs malades, aux femmes enceintes, aux nouveaux parents ou aux personnes s'occupant d'un membre de leur famille qui est gravement malade).

\section{Portraits de femmes}

Les propos recueillis auprès des femmes elles-mêmes et des intervenants soulignent à quel point les femmes judiciarisées avaient vécu et vivaient encore de multiples problèmes qui fragilisaient, freinaient, voire empêchaient toute tentative d'un retour à l'emploi après la sortie. Par exemple, la majorité des femmes détenues dans les établissements fédéraux et provinciaux ont connu ou connaissent encore divers problèmes de dépendance aux drogues.

Toujours selon les femmes interviewées dans le cadre de notre étude, outre la toxicomanie, les problèmes de santé et particulièrement de santé mentale, le fait de bénéficier depuis longtemps d'allocations d'assistanceemploi, le recours plus ou moins long à des activités illégales caractérisent aussi leur profil. Ce qui est aussi documenté dans d'autres études (Trevethan, 1999; Frigon et Duhamel, 2006).

Plusieurs femmes interviewées signalent en outre avoir connu un passé lourd d'abus physiques, sexuels ou psychologiques: "J'ai été une enfant violentée, j'ai été une enfant abusée. Je ne suis pas responsable d'avoir été abusée à sept ans » (Héloïse, incarcérée en prison provinciale, 42 ans). Ce passé place beaucoup de femmes dans une logique de victimisation et de souffrance qui les affecte définitivement. «Les filles, je trouve qu'elles ont comme des couches de souffrance qui s'empilent» (Stéphanie, intervenante au provincial).

Arrivées en prison, beaucoup de femmes perdent le contact avec leurs proches et se trouvent démunies affectivement et matériellement à leur sortie: 
Et puis, quand tu arrives dehors, tu as tout perdu: tu es toute seule, tu as perdu ton logement, tu as perdu tes meubles, tu as tout perdu. Tu n'as pas d'adresse, tu n'as pas de bien-être, tu n'as pas de BS [acronyme utilisé familièrement et désignant le «bien-être social», ancienne dénomination de l'assistance-emploi]. Tu n'as pas de BS, tu n'as pas d'adresse. Il faut que tu recommences tout. (Roxy, incarcérée en prison provinciale, 38 ans)

Pour ces personnes qui ont accumulé des «couches de souffrance» au cours de leur existence, on comprend alors que la question de se trouver un travail reste une préoccupation secondaire par rapport à l'ensemble des autres problèmes auxquels elles ont à faire face (reprendre la garde des enfants, trouver un logement, se faire soigner).

Pour beaucoup d'entre elles, l'emploi ne semble donc pas revêtir une importance aussi significative que la relation avec les enfants et avec les proches. C'est un «mal nécessaire», une obligation pour gagner sa vie: «Pour moi, la réinsertion sociale, c'est vraiment ma vie, ça tourne autour de mes enfants et ça, c'est ma priorité» (Isabelle, sous surveillance en maison de transition, 35 ans).

Pour certaines interviewées, malgré les autres défis à relever après la sortie, avoir un travail constitue une occasion de construire ou de reconstruire la confiance en elles qu'elles ont perdue:

Avoir un travail qui va pouvoir m'aider à redevenir confiante en moi-même. Aussi longtemps que je n'aurai pas quelque chose de concret dans ma vie, je vais trouver que je n'ai pas confiance en moi-même. (Sheila, sous surveillance en maison de transition, 44 ans)

Comme pour d'autres groupes marginalisés, certaines femmes interviewées soulignent aussi à quel point les emplois auxquels elles peuvent avoir accès ne permettent pas vraiment d'améliorer leur situation, surtout quand les profits de leurs anciennes activités illégales leur permettaient de vivre plus confortablement. De plus, certains métiers disponibles sont peu intéressants (difficultés de transport, de garde des enfants, salaires trop bas et mauvaises conditions de travail), ce qui pousse bien des femmes à rester bénéficiaires d'allocations d'assistance-emploi.

Tout comme l'étude dirigée par Combessie (2005), notre recherche montre que trois fonctions sont assignées au travail carcéral: rémunératrice, occupationnelle et prospective. Le travail en détention est envisagé par les femmes comme un moyen d'accumuler de l'argent et aussi un moyen de fuir l'ennui et l'inactivité: 
Quand tu es à la cuisine, tu ne vois pas le temps passer, tu as une heure de passée, on dirait que ça ne fait rien que dix minutes que tu es là. Ça va vite, c'est le fun. (Cindy, incarcérée en prison provinciale, 37 ans)

Le travail a aussi une fonction prospective dans la logique de réinsertion car la participation au travail peut être un gage d'un réaménagement de la sentence et des conditions de libération conditionnelle. Selon Combessie et al. :

En effet, via le travail pénal, la détenue rémunérée peut, grâce à ses revenus, même modestes, commencer à payer les dommages causés aux victimes ou les amendes, et, avec son volontarisme au travail, elle présente, dit-on, de bons «gages de réinsertion». (Combessie et al., 2005: 102)

Malgré tout, le fait que le travail en prison soit peu payé reste un sujet de frustrations à cause des différents taux salariaux pratiqués au sein des ateliers. Angela, incarcérée en pénitencier fédéral, fait remarquer que certains ateliers attirent plus de femmes parce qu'elles y sont mieux payées, mais que ces activités sont peu valorisantes et peu formatrices. De plus, la reconnaissance des formations faites en prison est difficilement transférable à l'extérieur car elle est identifiée au milieu carcéral.

Ces propos rejoignent nombre de critiques faites au travail en milieu carcéral. Le travail carcéral a été présenté par ses partisans comme un outil efficace de réinsertion sociale, permettant aux personnes détenues d'acquérir des compétences professionnelles en favorisant aussi le développement personnel (Gillis, 2000). D'autres recherches plus critiques ont au contraire souligné son caractère peu formateur (Strimelle et Poupart, 2004). En ce qui concerne les femmes, elles sont encore souvent cantonnées à des tâches conventionnelles, stéréotypées: ménage, lavage, repassage, cuisine, couture, ce qui ne leur permet pas vraiment de prétendre à des emplois mieux rémunérés ou plus spécialisés une fois qu'elles sortiront.

Après avoir détaillé le profil des femmes judiciarisées, leur perception du travail et leur profil en emploi, abordons maintenant l'axe 2, consacré aux enjeux de l'intervention en matière d'employabilité.

\section{Les défis de l'intervention en emploi auprès des femmes judiciarisées}

Dans cette section, nous avons privilégié le discours des intervenants qui ont abordé et ciblé directement la question de l'intervention tandis 
que les femmes l'ont, pour leur part, moins abordée. Les propos des intervenants ont, malgré tout, relayé et traduit certaines des préoccupations centrales des femmes.

Malgré une plus grande préoccupation à l'endroit de l'amélioration des compétences en emploi des femmes judiciarisées, plusieurs intervenants rencontrés déplorent encore le manque de ressources disponibles à la sortie. S'ajoutent à cela des problèmes plus personnels propres à bien des femmes judiciarisées: le manque de confiance en soi, le manque de formation et d'expérience en emploi, autant de caractéristiques qui peuvent entraver leur motivation à s'en sortir. Pour les femmes ayant déjà exercé un emploi avant l'emprisonnement, les expériences varient suivant les personnes. La plupart d'entre elles ont eu des emplois peu qualifiés et plutôt précaires ${ }^{5}$.

Les intervenants remarquent aussi que les femmes judiciarisées se connaissent peu elles-mêmes, elles ignorent, exagèrent ou minimisent l'étendue de leurs compétences réelles. En vue de les aider à se trouver un travail, l'intervention en matière d'employabilité doit donc viser à faire naître chez elles une reconnaissance de leurs compétences propres et une connaissance du marché du travail actuel: «Le but, c'est de les faire sortir, les faire travailler, leur prouver qu'elles sont capables» (Manon, intervenante au provincial).

Pourtant, certaines études menées auprès des femmes en libération conditionnelle soulignent que, dans l'ensemble, les femmes sont plus nombreuses que les hommes à terminer cette période de libération sans récidive ou bris de conditions (Cyrenne, 2002: 49).

Les intervenants trouvent aussi important de montrer aux femmes les aspects positifs reliés au fait d'avoir un travail:

C'est que le travail n'amène pas juste de l'argent, il amène de la valorisation, un réseau social, une mobilisation, de la stimulation intellectuelle... C'est ça, mais elles ne le voient pas. C'est ça, le défi. (Daphnée, intervenante au fédéral)

Parmi leurs points forts en matière d'employabilité, les intervenants soulignent que les femmes sont capables de minutie, de précision. Quand elles sont motivées, elles s'investissent beaucoup dans ce qu'elles font: «Je pense qu'elles aiment le travail bien accompli, elles sont soucieuses

5. En 2003 , environ $40 \%$ des femmes canadiennes occupaient des «emplois précaires» par rapport à $30 \%$ chez les hommes (Towson, 2005: 4). 
de ce qu'elles font quand elles le font [...]. Du travail bâclé, c'est rare» (Johanne, intervenante au fédéral).

Le fait que les femmes expriment plus facilement leur vécu, leurs souhaits, leurs projets peut aussi aider à mieux les orienter et à trouver des activités qui correspondent mieux à leurs demandes. Leur ouverture d'esprit, leur capacité à se remettre en question et à suivre des formations sont aussi considérées comme des forces.

Les intervenants ont beaucoup à dire sur les différents aspects de leur travail d'intervention. Sonia souligne notamment l'importance d'aller au-delà du délit dans la compréhension de la personne incarcérée. L'intervention comporte plusieurs facettes et se fait sur différents plans. L'intervention en détention a ses propres défis. Par exemple, le travail auprès de la clientèle incarcérée est modulé par la notion de sécurité, ce qui entraîne inéluctablement des limites. On insiste à maintes reprises sur la préparation à la sortie et l'importance du suivi après la détention.

L'intervention auprès des femmes judiciarisées devient plus difficile lorsqu'elles quittent leur lieu de détention et sont suivies en communauté:

La plupart du temps, on a bien de la difficulté à les joindre à l'extérieur. Soit qu'elles n'ont pas d'adresse, soit qu'elles ont déménagé à plusieurs reprises, ça dépend. (Sonia, intervenante au provincial)

En général, lors de l'intervention, la question de l'emploi n'est pas souvent abordée directement par les femmes. Il faut privilégier une approche globale qui tienne compte des autres problèmes des femmes avant d'aborder la question de l'emploi:

C'est sûr que de travailler avec les femmes, c'est différent, les femmes vont communiquer plus, disons qu'il y a beaucoup plus d'émotions. C'est beaucoup plus long, une entrevue avec une femme, c'est beaucoup plus long. Je ne veux pas dire par là que les femmes ont des problèmes plus ou moins graves que les hommes, ce n'est pas ça, c'est que c'est plus long. (Darianne, intervenante au fédéral)

L'intervention varie également selon les différentes catégories d'âge. Pour les femmes plus âgées, l'emploi n'est pas nécessairement une priorité (Strimelle, 2007). Avec les plus jeunes qui restent longtemps en détention, il est possible d'entreprendre un travail plus assidu de formation et d'éducation. 
La durée de la sentence va aussi avoir un impact sur l'intervention en détention. Comme le souligne Charles, intervenant au fédéral, les femmes qui ont une sentence de courte durée n'osent pas s'investir dans une démarche de réorientation de carrière. Pour la clientèle détenue en prison provinciale, le phénomène des portes tournantes ${ }^{6}$ peut aussi affecter l'efficacité de l'intervention.

La question de la dépendance affective a aussi été identifiée comme une entrave possible:

Il y a des femmes qui sont très dépendantes de leur conjoint [...]. On monte un bon projet, puis elles se font un copain ou elles reprennent avec leur copain qui est de l'autre côté. Elles vont alors plus facilement tout laisser tomber. (Stéphanie, intervenante au provincial)

Dans leur pratique, les intervenants ont précisé qu'ils adaptaient leur type d'intervention selon le profil général des femmes et divisaient ce groupe en trois catégories distinctes: les femmes qui n'ont jamais travaillé, celles qui ont déjà exercé un travail légal et celle qui ont fait un travail illégal.

Pour ces dernières, l'intervention doit particulièrement tenir compte des avantages matériels liés à ces emplois, avantages que les femmes préfèrent conserver plutôt que de s'engager dans des activités plus légales mais nettement moins rémunératrices. Certaines femmes utilisent le travail du sexe comme un "à côté» qui leur permet de couvrir certaines dépenses. Selon les intervenants, on doit reconnaître cette réalité sans nécessairement l'accepter. Il faut prendre les femmes comme elles sont et les mener ensuite graduellement à d'autres solutions. Toutefois, l'attrait pécuniaire demeure souvent très fort pour les femmes qui ont exercé le travail du sexe ou toute autre activité illégale et qui ne pourraient arriver à mener un train de vie similaire en occupant des emplois plus conventionnels.

Même si certaines auteures et groupes analysent le travail du sexe justement comme un travail (Thiboutot, 2001 ; Parent, 1994; Parent et al., 2003 ; ConStellation, 2005), les intervenants ont du mal à accepter et valider cette approche. Ils préfèrent voir comment réutiliser ou transformer les aptitudes développées dans le cadre du travail illégal en les appliquant au domaine du travail légal: "Je demande toujours:

6. Désigne les personnes qui cumulent les petites sentences en prison, alternant brèves périodes de liberté et courtes sentences. 
Qu'est-ce que tu faisais? Comment t'y prenais-tu? Comment ça marchait? Au lieu de vendre ton corps, qu'est-ce que tu aimerais faire?» (Carole, intervenante au fédéral).

Ils reconnaissent néanmoins que les exigences du marché du travail sont tellement élevées pour toutes les femmes souvent sans expérience de travail, sans éducation, avec un casier judiciaire, que certaines d'entre elles n'ont d'autre choix que de retourner à leurs anciennes activités illégales:

Ce n'est pas facile sur le marché de l'emploi. Elles ont toutes sortes de difficultés: la $\mathrm{DPJ}^{7}$ sur le dos, un conjoint violent, les dettes et elles n'ont jamais travaillé. Imaginez comment la marche est haute! (Huguette, intervenante en maison de transition)

D'après les intervenants, l'intervention en emploi doit se mener différemment quand elle s'adresse aux femmes. Or, pendant longtemps, les besoins des femmes ont été assimilés à ceux des hommes en matière d'emploi. Selon certains intervenants que nous avons rencontrés et qui travaillent aussi avec une clientèle masculine, les hommes auraient des objectifs de travail plus réalistes et plus de possibilités de trouver des emplois. Par ailleurs, on peut penser que ce sont encore majoritairement les femmes qui doivent prendre en charge tout ce qui concerne la gestion matérielle du quotidien (enfants, ménage, etc.), elles ont donc moins de temps et d'énergie pour se consacrer exclusivement à la quête d'emploi. Certains intervenants ont mentionné que les femmes avaient une approche plus globale de la vie, ce qui les fragilisait, car lorsqu'un domaine de leur vie était ébranlé, tous les autres aspects étaient aussi affectés.

La nécessité de mentir sur son expérience carcérale, le besoin de masquer son ancienne identité auprès des nouveaux collègues et employeurs sont aussi des éléments qui rendent difficiles le retour dans la société et l'accès au marché du travail:

Il y a des filles qui vont me demander: «Est-ce que ça se voit que je suis une prostituée? Est-ce que ça se voit que j'ai le VIH? Si tu me voyais dans la rue, que penserais-tu de moi?» (Stéphanie, intervenante au provincial)

Celles qui réussissent à trouver un emploi nourrissent la crainte d'être découvertes et donc d'être rejetées, ce qui les fragilise et les oblige à camoufler leur passé et leur identité réelle. Certaines prennent néanmoins le risque de révéler leur statut d'«ex-détenue»:

7. Direction de la protection de la jeunesse. 
C'est un peu énervant. Quand ils m'ont demandé: «Avez-vous un casier judiciaire?» J'ai regardé la patronne et j'ai un peu souri, j'ai souri parce que j'étais très nerveuse. Puis j'ai expliqué mon histoire et pourquoi, pour qu'elle sache que ce n'était rien de mal. Elle [ma patronne] m'a gardée quand même, elle n'a pas porté de jugement. (Louise, sous surveillance en maison de transition)

Certains intervenants se plaignent aussi du scepticisme de certains collègues (hommes et femmes) par rapport à la réinsertion des femmes incarcérées. Pour ceux-ci, travailler auprès des femmes, «ça gruge»; les femmes judiciarisées sont considérées comme plus «difficiles»:

Il $\mathrm{y}$ a des fois où je me rends compte que les gens sont beaucoup plus sévères avec elles. Je me rends compte aussi qu'il y a des intervenants qui sont sévères. (Stéphanie, intervenante au provincial)

En quoi ces femmes sont-elles plus difficiles? Cette sévérité est-elle le fait de certaines catégories d'intervenants? S'exerce-t-elle dans des contextes particuliers? S'agit-il d'une catégorie spéciale de détenues jugées plus difficiles ou d'un stéréotype accolé à l'ensemble des femmes incarcérées? L'existence de pratiques discriminatoires à l'égard des femmes détenues a été signalée et dénoncée à plusieurs reprises (Faith, 2002; Carlen, 1983; Pate, 2002). Certaines y voient la permanence de stéréotypes sexistes et patriarcaux liés à l'image de la «femme délinquante»(Carlen,1983). Les intervenants que nous avons rencontrés n'ont pas approfondi cet aspect dans leurs propos et nous ne pouvons donc aller plus avant dans nos conclusions à ce sujet, on peut néanmoins s'interroger sur la pertinence de telles attitudes et sur les effets préjudiciables qu'elles peuvent avoir sur les femmes, notamment en matière d'aide à la réinsertion.

Nous venons de souligner à quel point l'approche d'aide à l'emploi différait selon la clientèle, le genre et l'expérience de travail. Malgré les difficultés identifiées par les intervenants, les femmes semblent bien accueillir les services spécialisés en matière d'employabilité et de maintien en emploi.

Nous abordons maintenant le dernier axe consacré aux pistes prometteuses, examinant les ressources, projets et changements souhaités dans l'approche préconisée auprès des femmes judiciarisées. 


\section{Pistes prometteuses en emploi}

Quelles sont les ressources pouvant aider les femmes à leur sortie? Comment pourrait-on améliorer l'approche d'aide à l'emploi pour les femmes judiciarisées?

Huguette, intervenante en maison de transition, souligne dans ses propos la nécessité de sensibiliser les employeurs aux demandes des femmes judiciarisées et de créer des liens significatifs avec la communauté pour assurer une meilleure transition.

Peu de femmes interviewées ont abordé cette question des ressources à l'extérieur. Certaines, ayant un réseau de soutien présent à la sortie, n'ont pas envisagé spécifiquement la question, tout en disant redouter un peu d'affronter ce retour dans le monde. D'autres ont manifesté la volonté d'avoir recours à diverses ressources pour se connecter à nouveau avec le monde du travail comme les centres de recherche d'emploi destinés aux personnes judiciarisées qui les mettent plus à l'aise:

J'ai plus de chances de me sentir moins rejetée, je vais me sentir plus à mon aise avec eux qu'avec un monsieur du BS et commencer à lui dire que j'ai seulement un secondaire 3 (troisième année du cycle secondaire) puis la prostitution puis la prison dans le corps, là. Tandis qu'au groupe $\mathrm{X}$ (organisme d'aide à l'emploi pour les personnes judiciarisées), ils sont habitués à ça. (Cindy, incarcérée en prison provinciale, 37 ans)

Les priorités des femmes touchent à la question des enfants et du logement (Rapport de la vérificatrice générale du Canada [RVGC], 2003 : 20). Parmi les projets pilotes favorisant le retour dans la communauté, Colette, intervenante au fédéral, est favorable aux appartements supervisés pour les femmes en libération conditionnelle. Elle souligne à quel point il serait important de prendre en compte la question du logement pour aider les femmes à aborder plus rapidement une démarche de recherche d'emploi.

Plusieurs autres éléments peuvent encourager l'employabilité et le maintien en emploi. Par exemple, lorsque la femme se sent valorisée et qu'elle réussit à franchir des étapes: «Il faut absolument que je sois valorisée dans ce que je fais. Il faut que je sois fière de moi, il faut que ça me valorise. Sinon je ne garde pas mes jobs» (Édith, incarcérée en prison provinciale, 33 ans).

La formation offerte en prison est un élément à prendre en considération pour encourager l'employabilité: 
C'est beaucoup aller coudre des bobettes, je pense que ce n'est pas valorisant, que ce n'est pas une chose qui va leur servir à la sortie [...]. Malheureusement, les filles vont là où il $\mathrm{y}$ a plus de sous, elles vont donc aller coudre des bobettes au lieu d'aller à l'école [...]. Pourquoi ne pas donner un bonus pour qu'elles aillent à l'école? (Isabelle, sous surveillance en maison de transition, 35 ans)

Nombre de remarques faites par les intervenants et aussi par les femmes vont dans le sens des recommandations faites en 2003 par la vérificatrice générale du Canada. Dans le chapitre de son rapport annuel consacré à la réinsertion sociale des délinquantes, elle recommandait au service correctionnel du Canada d'élaborer une stratégie à l'intention des femmes en vue d'améliorer leurs compétences dans des domaines en demande sur le marché de l'emploi. Elle soulignait aussi la nécessité d'un suivi plus intensif pendant les six premiers mois suivant la sortie de prison:

Certaines doivent absolument obtenir l'aide et le soutien nécessaires à ce moment précis, car c'est ce qui pourrait leur permettre de retrouver une certaine maîtrise de leur vie et de ne pas revivre les conditions qui ont été à l'origine de leur comportement criminel. (RVGC, ch. 4, 2003: 22)

La vérificatrice encourageait aussi la multiplication de lieux d'hébergement pour les femmes à leur sortie d'incarcération et le développement de ressources spécifiques aux femmes autochtones, aux femmes souffrant de problèmes de santé mentale et de toxicomanie. Elle évoquait enfin le besoin de mettre les délinquantes en relation avec des ressources dans la collectivité avant la sortie de prison, ce qui leur permettrait d'établir un réseau de relations dont elles auraient besoin au moment de leur libération.

Actuellement, on peut noter plusieurs initiatives dans le milieu carcéral ou communautaire au Québec pour favoriser la prise en charge par les femmes de leurs responsabilités parentales (programme Mèreenfant à Joliette et Continuité famille à la Maison Tanguay), le développement de leurs compétences en matière éducative et en formation professionnelle (entrée d'organismes d'aide à l'emploi OPEX'82 dans les établissements Tanguay, Joliette et La Jonction à Québec). Certaines initiatives (programme canin à Joliette; art-thérapie à Tanguay) sont aussi marquées par le désir de développer une approche plus globale en matière d'aide aux femmes. Le recours à ces nouvelles stratégies vise à assurer une prise en charge qui tienne compte de toutes les facettes 
de la vie des femmes et à développer certaines aptitudes ignorées jusqu'alors, comme la créativité.

Néanmoins, ces initiatives restent encore trop ponctuelles pour assurer à toutes les femmes une aide effective à la sortie de prison et garantir une réinsertion réussie.

\section{Conclusion}

Notre article a été consacré à la question du travail des femmes judiciarisées afin de mieux comprendre comment se pose la question de l'emploi pour elles et le sens qu'elles lui accordent. Est-ce que le fait de trouver un emploi ou de garder un emploi est une condition indispensable pour ne pas «retomber» dans la délinquance et se retrouver en prison? Pour ces femmes, l'intégration sociale passe-t-elle nécessairement par l'obtention d'un emploi?

Comme nous l'avons vu au cours de cet article, les initiatives visant à favoriser la réinsertion sociale des femmes judiciarisées commencent à se développer depuis dix ans. Or, durant cette même période, on observe parallèlement une augmentation croissante du nombre de femmes incarcérées (RVGC, 2003 : 5; Pate, 2002: 148).

La question suivante se pose alors: comment s'assurer que de telles initiatives rempliront leur objectif de réinsertion alors que de plus en plus de femmes auront à faire face aux multiples défis du retour dans la société et à subir les conséquences stigmatisantes de leur séjour en milieu correctionnel, particulièrement quand elles seront en recherche d'emploi? Plutôt que d'avoir recours plus fréquemment à l'emprisonnement des femmes, ne serait-il pas plus opportun d'utiliser des alternatives à l'enfermement ou de sortir les femmes de prison le plus tôt possible en leur assurant une aide et une supervision effectives afin de faire l'économie d'un appareil répressif?

D'autre part, aborder la question du retour au travail après la prison porte aussi à s'interroger plus largement sur les ressources que le système pénal et que les mesures de protection sociale offrent aux personnes sorties d'incarcération. Depuis les années 1980, et la remise en question de «l'État social», les mesures d'aide sociale et d'aide à l'emploi ont été considérablement réduites et, même si les individus en situation précaire peuvent encore avoir accès à certaines ressources et certaines formes d'aide, le message que leur transmet le système de protection sociale est 
qu'ils doivent être capables de s'en sortir par eux-mêmes (Otero et al., 2004). La logique du système pénal repose aussi de plus en plus sur l'idée que les personnes judiciarisées doivent porter la responsabilité de leur propre réinsertion (Strimelle et Poupart, 2004; Ehrenberg, 1998). Comment les femmes judiciarisées peuvent-elles vivre toutes ces contraintes et porter le poids de leur propre réinsertion sans devenir encore plus vulnérables? Suffit-il d'être de «bonne volonté» pour s'en sortir? Comment penser et agir sur la criminalisation du travail du sexe? Les intervenants rencontrés n'ont pas vraiment soulevé ces problèmes, mais d'après nous, ces questions doivent être posées.

Tout ceci porte enfin à s'interroger sur l'efficacité des formes d'intervention pénales auprès des femmes et la persistance d'une logique carcérale qui continue à s'imposer malgré les multiples échecs qu'on lui reconnait. D'ailleurs, pour certaines chercheures féministes, l'emprisonnement ne peut en aucun cas répondre de façon appropriée aux besoins des femmes judiciarisées, la prison restant à leurs yeux un bastion de la culture masculine (Faith, 2002: 125). En ce sens, notre recherche souligne la nécessité de repenser les formes d'intervention auprès des femmes judiciarisées en matière d'emploi, en prêtant attention à la singularité de leurs expériences, en tenant compte du contexte structurel d'oppression qu'elles subissent, en écoutant ce qu'elles ont à nous dire sur leurs besoins par rapport à l'emploi, en essayant enfin d'atténuer, voire de supprimer l'idéologie répressive dont les effets peuvent empêcher parfois définitivement toute tentative de réinsertion.

\section{Références}

Bertrand, M.-A. (2002). Progrès, recul et stagnation: Tableau contrasté des conditions de vie des femmes incarcérées au Canada. Criminologie, 35 (2), 135-146.

Bertrand, M.-A. (2003). Les femmes et la criminalité. Montréal: Éditions Athéna. Blanchard, B. (2002). La situation des mères incarcérées et de leurs enfants au Québec. Criminologie, 35 (2), 91-112.

Bloom, B. (1999). Les programmes conçus spécialement pour les délinquantes: Principes directeurs et pratiques. Forum/ Recherche sur l'actualité correctionnelle, 11 (3), 22-27.

Brouillard, J., \& Sirois, A. (1996). L'employabilité en pratique, Forum/Recherche sur l'actualité correctionnelle, 8 (1), 32-34.

Buck, M. (2004). Women in Prison and Work. Feminist Studies, 30 (2), 451455. 
Carlen, P. (1983). Women's Imprisonment: A Study in Social Control. Londres: Routledge.

Castel, R. (1994). La dynamique du processus de marginalisation: de la vulnérabilité à la désaffiliation. Cabiers de recherche sociologique, 22, 11-27.

Castel, R. (1995). Les métamorphoses de la question sociale. Une chronique du salariat. Paris: Fayard.

Combessie, P. (2004). Intégration sociale des anciens détenus. Analyse des logiques de la justice pénale et de leurs effets. In J. Poupart (Dir.), Au-delà du système pénal. L'intégration sociale et professionnelle des groupes judiciarisés et marginalisés (231-248). Sainte-Foy: Presses de l'Université du Québec.

Combessie, P. (Dir.) (2005). Femmes, intégration et prison: analyse des processus d'intégration socioprofessionnelle des femmes sortant de prison en Europe. Women, Integration and Prison: an Analysis of the Processes of Socio-Labor Integration of Women after Prison in Europe. Paris: FAIRE.

Comité aviseur pour la clientèle judiciarisée adulte (2001). La formation des personnes judiciarisées. Montréal: Comité aviseur pour la clientèle judiciarisée adulte.

ConStellation (2005). Spécial prison/Prison Special, 9 (1).

Cyrenne, M-A. (2002). Réflexions sur la réalité actuelle des détenues dans le système correctionnel fédéral. Criminologie, 35 (2), 31-52.

Ehrenberg, A. (1998). La fatigue d'être soi. Dépression et société. Paris: Éditions Odile Jacob Poche.

Faith, K. (2002). La résistance à la pénalité: un impératif féministe. Criminologie, 35 (2), 113-134.

Faugeron, C. (1996). Introduction: une théorie de la prison est-elle possible? In C. Faugeron, A. Chauvenet \& P. Combessie (Eds.), Approches de la prison (15-41). Paris, Bruxelles: De Boeck Université.

Foucault, M. (1975). Surveiller et punir. Naissance de la prison. Paris: Gallimard.

Frigon, S. (2002). La création de choix pour les femmes incarcérées: sur les traces du groupe d'étude sur les femmes purgeant une peine fédérale et de ses conséquences. Criminologie, 35 (2), 9-30.

Frigon, S. (2004). Transformation de la philosophie et de la gestion pénales des femmes justiciables au Canada: trois cas de figure. Femmes et Droit, 16 (2), 353-385.

Frigon, S., Strimelle V., \& Renière, C. (2003). Insertion et maintien en emploi des femmes judiciarisées au Québec. Montréal: Comité aviseur pour la clientèle judiciarisée adulte.

Frigon, S., \& Duhamel, N. (2006). Regards croisés sur la santé mentale des femmes avant, pendant et après l'incarcération. Ottawa: Condition féminine Canada.

Garland, D. (1985). Punishment and Welfare: A History of Penal Strategies. Hants: Gower Publishing.

Gillis, C. (1996). L'emploi des détenus: l'importance croissante des compétences génériques. Forum/Recherche sur l'actualité correctionnelle, 12 (2), 18-20.

Gillis, C. (2000). Repenser l'emploi des délinquants. Forum/Recherche sur l'actualité correctionnelle, 12 (2), 32-35. 
Hannah-Moffat, K. (2001). Punishment in Disguise: Penal Governance and Federal Imprisonment of Women in Canada. Toronto: The University of Toronto Press.

Laberge, D., \& Roy, S. (1994). Marginalité et exclusion sociales: des lieux et des formes. Cabiers de recherche sociologique, 22, 4-9.

Lalande, P. (2000). Évolution des politiques pénales et du discours à propos de l'emprisonnement au Canada et au Québec de 1969 à 1999. Québec: Direction générale des services correctionnels.

Otero, M., Poupart, J., \& Spielvogel, M. (2004). Sortir et s'en sortir. La montée de la norme d'autonomie dans les trajectoires de retour à la communauté des «ex-détenus». In J. Poupart (Dir.), Au-delà du système pénal. L'intégration sociale et professionnelle des groupes judiciarisés et marginalisés (61-82). Sainte-Foy: Presses de l'Université du Québec.

Parent, C. (1994). La «prostitution» ou le commerce des services sexuels. In F. Dumont, S. Langlois, \& Y. Martin (Dir.), Traité des problèmes sociaux (393409). Québec: Institut Québécois de recherche sur la culture.

Parent, C., Bruckert, C., \& Robitaille, P. (2003). Établissements de services érotiques/danse érotique: deux formes de travail marginalisé. Ottawa: Commission de réforme du droit.

Pate, K. (2002). Recension des dix dernières années relativement aux femmes condamnées à des peines fédérales: de mal en pis. Criminologie, 35 (2), 147158.

Paugam, S. (1991). La disqualification sociale: essai sur la nouvelle pauvreté, Paris: Presses universitaires de France.

Paugam, S. (1993). La société française et ses pauvres. Paris: Presses universitaires de France.

Poupart, J., Dozois, J., \& Lalonde, M. (1989). Construction sociale de la dangerosité, pratique criminologique et système pénal. Rapport de recherche, École de criminologie: Université de Montréal.

Rapport de la vérificatrice générale du Canada à la Chambre des Communes (2003). Ottawa: Bureau du vérificateur général du Canada.

Richie, B. (2001). Challenges incarcerated women face as they return to their communities: findings from life history interviews. Crime \& Delinquency, 47 (3), 368-389.

Schmitz, F. (1985). Les effets du casier judiciaire sur la recherche d'emploi. Mémoire inédit de maîtrise en criminologie, Université de Montréal.

Schnapper, D. (1997). Contre la fin du travail. Paris: Éditions Textuel.

Service correctionnel du Canada (1990). La création de choix: Groupe d'étude sur les femmes purgeant une peine fédérale. Ottawa: Ministère des Approvisionnements et services.

Strimelle, V., \& Poupart, J. (2004). Une fois la sentence terminée. L'intégration des personnes ayant connu une expérience d'intégration. In J. Poupart (Dir.), Au-delà du système pénal. L'intégration sociale et professionnelle des groupes judiciarisés et marginalisés (101-123). Sainte-Foy: Presses de l'Université du Québec. 
Strimelle, V. (2007). À la recherche d'une population invisible: les femmes âgées en prison au Canada. Revue de Droit Pénal et de Criminologie, sous presse.

Thiboutot, C. (2001). Lutte des travailleuses du sexe: perspectives féministes. In Fédération des femmes du Québec. Rapport du comité de réflexion sur la prostitution et le travail du sexe (6-12). www.cybersolidaires.org/prostitution/ docs/ffq2.html

Towson, M. (2005). La problématique de la pauvreté pour les Canadiennes, document d'information. Ottawa: Condition féminine Canada.

Trevethan, S. (1999). Les femmes incarcérées dans les établissements fédéraux, provinciaux ou territoriaux. Forum/Recherche sur l'actualité correctionnelle, 11 (3), 9-12. 